\title{
Subretinal exudative deposits in central serous chorioretinopathy
}

Darmakusuma Ie, Lawrence A Yannuzzi, Richard F Spaide, Maurice F Rabb, Norman P Blair, Mark J Daily

Department of Ophthalmology, Manhattan Eye, Ear and Throat Hospital, New York

DIe

L A Yannuzzi

R F Spaide

Department of Ophthalmology and

Visual Sciences, UIC Eye Center, University of Illinois at Chicago

College of Medicine and

Mercy Hospital and

Medical Center, Chicago,

Illinois

M F Rabb

Laboratory of Retinal

Circulation and

Metabolism, Department

of Ophthalmology and

Visual Sciences, UIC Eye

Center, University of

Illinois at Chicago

College of Medicine,

Chicago, Illinois

N P Blair

Department of

Ophthalmology, Loyola

University Medical

School, Maywood,

Illinois

M J Daily

Correspondence to:

Dr L A Yannuzzi,

Manhattan Eye, Ear and

Throat Hospital, 210 East 64th

Street, New York, NY 10021,

USA.

Accepted for publication

26 January 1993

\section{Abstract}

The presence of subretinal exudation in a patient with neurosensory detachment of the macula frequently suggests the diagnosis of choroidal neovascularisation. A retrospective chart review of newly diagnosed cases of central serous chorioretinopathy revealed 11 patients, seven men and four non-pregnant women, who had plaques of subretinal exudate, which presumably were fibrin. Each of these patients had a solitary plaque that ranged in size from 300 to $1500 \mu \mathrm{m}$ in diameter. These patients had no signs or a clinical course suggestive of choroidal neovascularisation. In each case the subretinal plaque was overlying an exuberant leak in the retinal pigment epithelium. The exudate was generally present at the initial examination, and usually showed dissolution before or coincident with the resolution of the neurosensory detachment. After resolution of the central serous chorioretinopathy, patients were left with subtle alterations in the retinal pigment epithelium in the areas of the subretinal plaque. These findings are important for two reasons. Firstly, the presence of subretinal exudation does not necessarily rule out the diagnosis of central serous chorioretinopathy. Secondly, pathophysiological theories of central serous chorioretinopathy must explain how the plaques are deposited behind the retina.

(Brf Ophthalmol 1993; 77: 349-353)

Neurosensory detachment of the macula can occur in adults for a variety of reasons; some conditions require prompt treatment while others can be expected to resolve spontaneously. The presence of subretinal exudative deposits may suggest the presence of choroidal neovascularisation. On the other hand, central serous chorioretinopathy, except in pregnant women $^{1}$ or cases of atypical large bullous detach-

Table 1 Patient characteristics

\begin{tabular}{|c|c|c|c|c|c|}
\hline Patient & $\begin{array}{l}\text { Age/sex/ } \\
\text { race }^{\star}\end{array}$ & $\begin{array}{l}\text { Initial visual } \\
\text { acuity }\end{array}$ & $\begin{array}{l}\text { Final visual } \\
\text { acuity }\end{array}$ & $\begin{array}{l}\text { Presence of } \\
R P E D \dagger\end{array}$ & $\begin{array}{l}\text { Laser } \\
\text { treatment }\end{array}$ \\
\hline $\begin{array}{r}1 \\
2 \\
3 \\
4 \\
5 \\
6 \\
7 \\
8 \\
9 \\
10 \\
11\end{array}$ & $\begin{array}{l}44 / \mathrm{M} / \mathrm{A} \\
35 / \mathrm{F} / \mathrm{W} \\
43 / \mathrm{M} / \mathrm{W} \\
38 / \mathrm{M} / \mathrm{W} \\
48 / \mathrm{F} / \mathrm{W} \\
47 / \mathrm{M} / \mathrm{W} \\
29 / \mathrm{F} / \mathrm{W} \\
44 / \mathrm{M} / \mathrm{H} \\
37 / \mathrm{M} / \mathrm{A} \\
40 / \mathrm{M} / \mathrm{A} \\
48 / \mathrm{F} / \mathrm{H}\end{array}$ & $\begin{array}{l}20 / 400 \\
20 / 25 \\
20 / 70 \\
20 / 100 \\
20 / 25 \\
20 / 60 \\
20 / 70 \\
20 / 200 \\
20 / 200 \\
20 / 80 \\
20 / 200\end{array}$ & $\begin{array}{l}20 / 70 \\
20 / 25 \\
20 / 200 \\
20 / 20 \\
20 / 25 \\
20 / 100 \\
20 / 20 \\
20 / 25 \\
20 / 40 \\
20 / 20 \\
20 / 30\end{array}$ & $\begin{array}{l}\text { Yes } \\
\text { Yes } \\
\text { Yes } \\
\text { Yes } \\
\text { Yes } \\
\text { Yes } \\
\text { Yes } \\
\text { No } \\
\text { Yes } \\
\text { Yes } \\
\text { Yes }\end{array}$ & $\begin{array}{l}\text { No } \\
\text { No } \\
\text { No } \\
\text { No } \\
\text { No } \\
\text { Yes } \\
\text { No } \\
\text { Yes } \\
\text { Yes } \\
\text { Yes } \\
\text { Yes }\end{array}$ \\
\hline
\end{tabular}

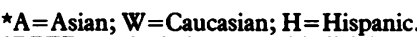

†RPED=retinal pigment epithelial detachment.

ments, ${ }^{2}$ are generally not associated with subretinal exudative deposits.

We evaluated a group of 11 patients with central serous chorioretinopathy who had subretinal exudative deposits for the following purposes: firstly, to identify non-pregnant women as another subgroup of central serous chorioretinopathy patients who develop subretinal exudative deposits; secondly, to describe the findings and clinical course of these patients more completely than in previous papers; thirdly, to define characteristics of subretinal exudative deposits that differentiate them from the typical exudate seen in choroidal neovascularisation; fourthly, to hypothesise a mechanism integrating results from previous studies by which subretinal exudative deposits form.

Patients were selected retrospectively from the files of the contributing authors. Central serous chorioretinopathy was defined as an idiopathic detachment of the neurosensory retina, associated with focal or multifocal leaks at the level of the retinal pigment epithelium demonstrable on fluorescein angiography. The patients had complete ophthalmic examinations, fundus colour photography, and fluorescein angiography. All patients were followed at least until the resolution of their primary detachments.

\section{Results}

The 11 patients included seven men and four women (Table 1). All were newly diagnosed and all were in good health and none of the women were pregnant. The mean age of the patients was 41 (SD 6) years with a range from 29 to 48 years. The mean age of the four women was 40 years, considerably older than the mean of age of 31 reported by Gass in his series of pregnant patients. ${ }^{1}$ Six were Caucasian, three were Asian, and two were Hispanic. Refractive errors were plano to +1.00 in all patients following resolution of the macular detachment except for one patient who was $-2 \cdot 25$. None of the patients had angioid streaks, peripheral punched out chorioretinal atrophic spots, peripapillary atrophy, chorioretinitis, or soft drusen.

In each case there was a neurosensory detachment associated with a solitary, focal, featheredged plaque on the undersurface of the retina (Fig 1). The placoid deposits were generally greyish-white to white, but were occasionally cream-coloured. These deposits were translucent to retroillumination. No patient had haemorrhage or cystoid macular oedema, which can be signs suggestive of choroidal neovascularisation. No patient had the bright, yellow white deposits under the retina characteristic of lipid. Confusion initially caused by the greyish- 
Figure 1(A) A 48-year-old woman with a history of reduced vision in the right eye of several days' duration. There is a neurosensory retinal detachment in the temporal macula overlying a yellowish deposit of exudate. In the inferonasal macula, there is another serous detachment of the retinal pigment epithelium.
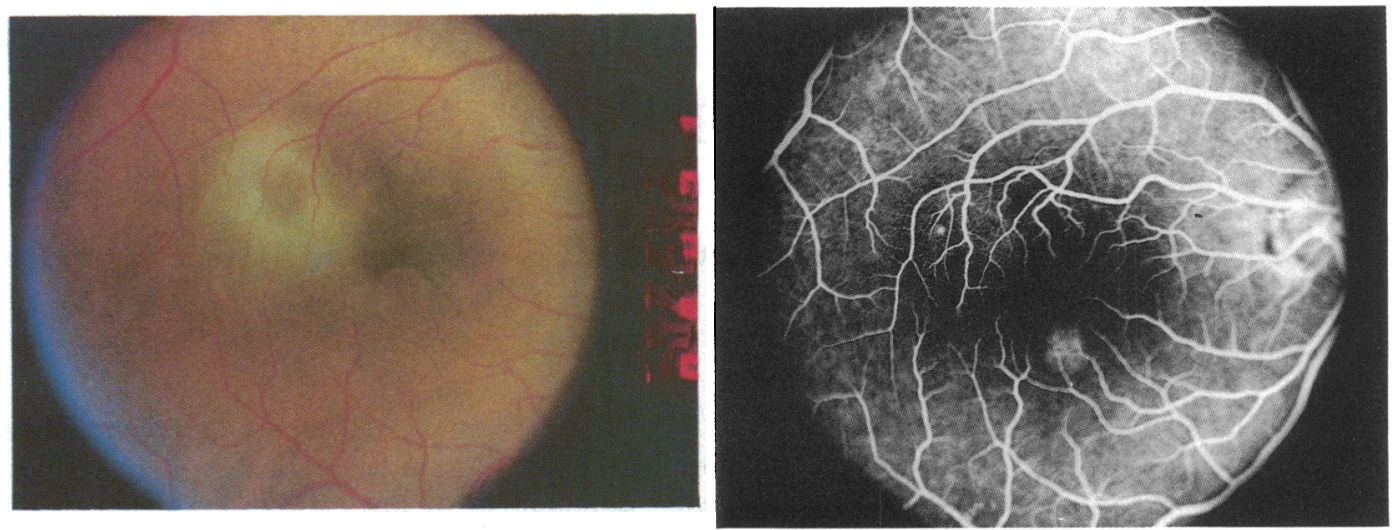

Figure $1(B)$ The early venous phase fluorescein angiogram reveals hyperfluorescence from pooling beneath the serous

white appearance of the plaques led to mistaken diagnoses of choroidal neovascularisation, retinitis, and, in one patient, an infiltrative metastatic process to the choroid. The subretinal exudative deposit was found between the arcades in 10 of the 11 patients; in one patient it was found nasal to the disc.

The exudate varied in size from $300 \mu \mathrm{m}$ to $1500 \mu \mathrm{m}$ in diameter. The size of the exudate did not seem to correlate with size of the detachment. In our cases, the subretinal exudative deposit often had a partial or complete ring appearance where the centre was thinner and more pellucid than the edges. The inferior portion of the exudate was usually thicker than the superior portion.

On fluorescein angiography the subretinal exudative deposit was consistently found directly above or adjacent to a robust leak from the level of the retinal pigment epithelium (Fig 2). The exudate did not block the underlying fluorescence. A retinal pigment epithelial detachment was found in 10 of the 11 patients, and the leak was almost always at or very near the edge of the retinal pigment epithelial detachment. In a few patients the ring of subretinal exudation encircled the retinal pigment epithelial detachment like a halo. No patient had evidence of retinal vascular leakage.

A majority of our cases had spontaneous resolution of their detachments in 3-6 months. Five patients were treated by laser photocoagulation (Fig 3). One author (LAY) followed three patients for more than 7 years. One of these patients experienced chronic and recurrent acute detachments with progressive perifoveal atrophy, cystic macular degeneration, and visual

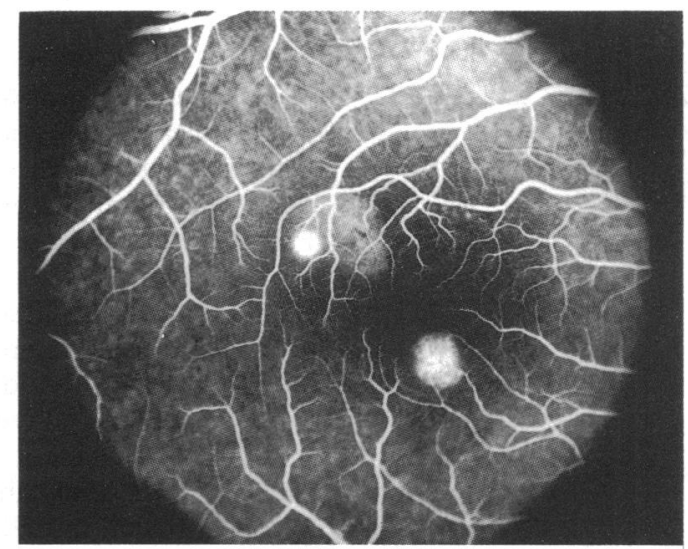
detachments of the retinal pigment epithelium. The superior pigment epithelial detachment is associated with a focal leak at its temporal margin.

decline to $20 / 100$. The other two patients, one followed for 8 and one for 9 years, demonstrated no recurrences and visual acuity recovery to 20/20. Nopatient in the series developed choroidal neovascularisation.

The dissolution of the exudate did not necessarily parallel the course of the macular detachment. It was usually present at the time of diagnosis. In some cases, the exudate disappeared long before the sensory retinal detachment resolved. In a few patients, the ring of exudate persisted surrounding a serous pigment epithelial detachment for a variable period after the resolution of the sensory retinal detachment. In others, the disappearance of the exudate coincided with the resolution of the sensory retinal detachment. Some patients were left with a subtle greyish discoloration in the area of the subretinal exudative deposit.

\section{Discussion}

The subretinal deposition described in this report appears to be a primary manifestation of central serous chorioretinopathy. Recognised secondary alterations in central serous chorio-

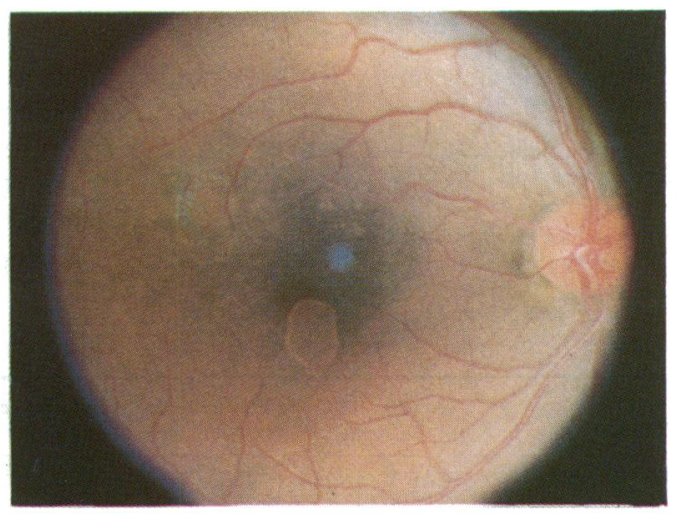

Figure 1(D) A photograph of the same patient following laser photocoagulation treatment to the pigment epithelial leak in the superior macula. There has been resolution of the neurosensory retinal detachment and flattening of the serous detachment of the retinal pigment epithelium in the superior The exudate which was surrounding the pigment epithelial detachment in the macula has also resolved, and the photocoagulation has left a slight atrophic defect at the treatment site. macula. The inferior pigment epithelial detachment persists. 
Figure 2(A) Colour photograph of a 44-year-old man with a history of change in vision of several days' duration. There is an exudative detachment of the neurosensory retina with an irregular area of subretinal yellowish exudate near the fovea.

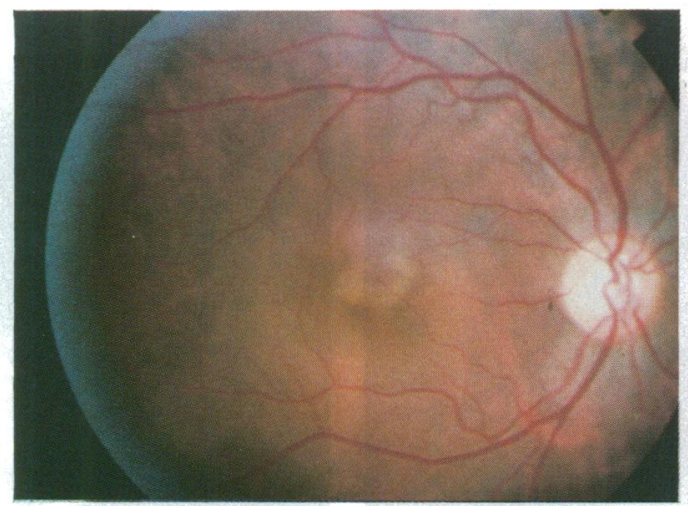

retinopathy include capillary telangiectasis, ${ }^{3}$ retinal capillary non-perfusion, ${ }^{34}$ retinal neovascularisation, ${ }^{4}$ lipid deposition, cystoid macular oedema, dependent retinal detachment, ${ }^{25}$ retinal pigment epithelial descending tracts, ${ }^{3}$ and choroidal neovascularisation. Most, if not all, of these changes represent sequela associated with chronic disease. They have not been associated with primary detachment of the macula in newly diagnosed cases such as the patients described in this series.

Some of the patients in this report were initially suspected of having choroidal neovascularisation because of the exudate. However, biomicroscopic examination of these patients did not reveal turbid subretinal fluid or haemorrhage. In addition, these patients did not have angioid streaks, chorioretinitis, or chorioretinal atrophic spots, which in young people are sometimes associated with choroidal neovascularisation. Furthermore, the clinical course, with improvement in signs and symptoms even without laser treatment, also was consistent with the diagnosis of central serous chorioretinopathy.

On fluorescein angiography, almost all of these patients had a retinal pigment epithelial detachment with a focal leak from its edge. The subretinal exudative deposit typically was a focal deposit that appeared to overlie the leak. 'Blow outs' of the retinal pigment epithelium have previously been described, ${ }^{6}$ but these 'blow outs' occurred on the surface of the retinal pigment epithelial detachment. It has been shown that the area of maximal stress is at the margin of a pigment epithelial detachment. ${ }^{7}$ It is possible that the patients described in this report have a 'subclinical blow out' of the retinal pigment epithelium at the border of the retinal pigment epithelial detachment, allowing the abnormal

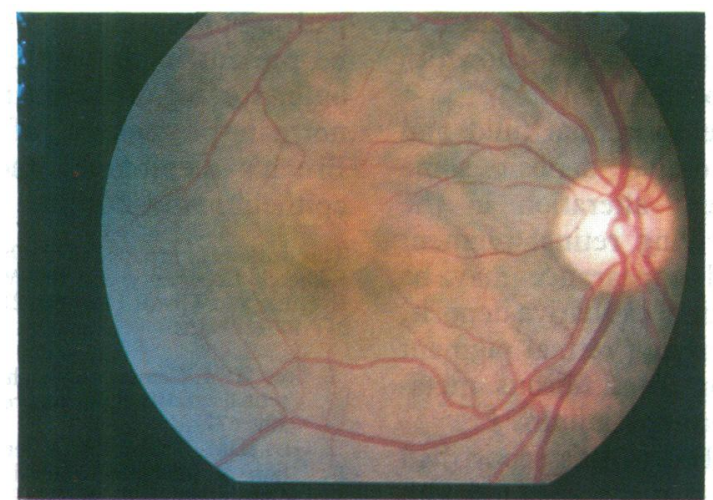

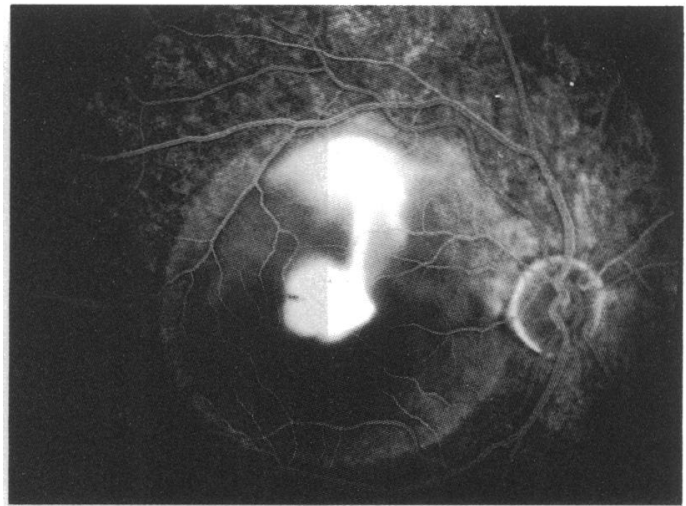

Figure 2(B) The late stage fluorescein angiogram reveals the presence of a serous detachment of the retinal pigment epithelium with overlying retinal pigment epithelial hyperplastic figures in the central macula. There is a 'smokestack' leak emanating from a point near or at the margin of the retinal pigment epithelium, ascending in the subneurosensory retinal space and decussating temporally more than nasally. Some of the subretinal fluid has been stained with fluorescein, more clearly delineating the full extent of the neurosensory detachment.

egress of fluid and protein under the retina. This phenomenon has recently been described in cases of age-related macular degeneration. ${ }^{8}$

The subretinal exudative deposition associated with central serous chorioretinopathy differed in character and location from that seen in choroidal neovascularisation. In acute stages of choroidal neovascularisation, the two most common materials deposited under the retina are blood and lipid. Blood is most commonly seen near the area of choroidal neovascularisation or along the dependent edge of the neurosensory detachment. Lipid is most commonly seen at the rim of the neurosensory detachment and is characterised by yellow white, hard-edged, opaque subretinal flecks. The deposits seen in the present series of patients were feather edged, greyish-white, translucent, and occurred in close proximity to the fluorescein leak.

The composition of the subretinal exudative deposits is not well established. The material did not appear to be lipid. The patients in this and a previous report ${ }^{1}$ did not appear to have systemic inflammatory disease or clinical evidence of ocular inflammation, making the possibility of subretinal white cell deposition unlikely. Histopathological and histochemical studies of one case of central serous chorioretinopathy has shown the presence of subretinal and subpigment epithelial fibrin. ${ }^{9}$ Histopathological analysis has revealed the presence of a high protein content within the subretinal fluid. ${ }^{10}$ This high concentration of protein may be the cause of smokestack leaks seen in central serous chorioretinopathy." These studies, as well as the clinical appearance of the deposits, suggest that there is proteinaceous material, including the possibility of fibrin, present in the subretinal space in central serous chorioretinopathy which might contribute to the formation of a subretinal exudative deposit.

Previous hypotheses regarding the pathogenesis of central serous chorioretinopathy have proposed a derangement of retinal pigment epithelial function as the initiating cause in central serous chorioretinopathy. ${ }^{12-14}$ The nature 


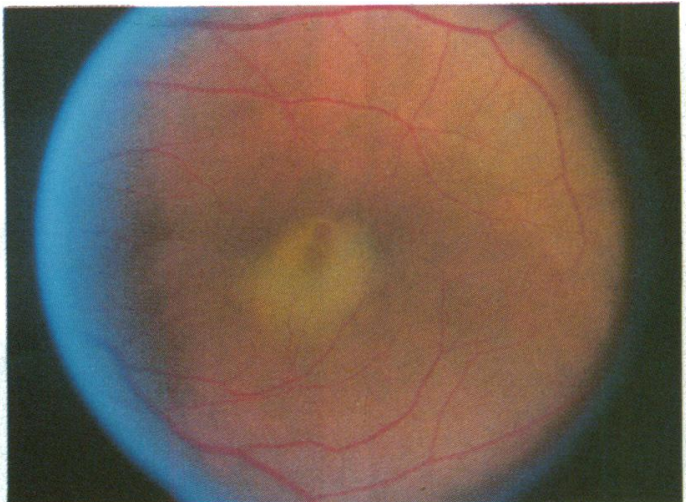

Figure 3(A) A colour photograph of a 40-year-old man with a I week history of reduced vision in the left eye. There is a neurosensory retinal detachment overlying a deposit of exudate which partially obscures a serous detachment of the retinal pigment epithelium.

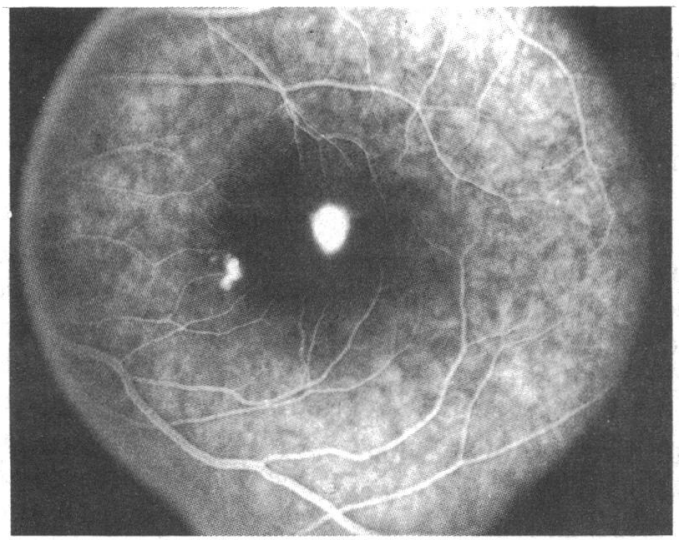

Figure 3(C) Late venous phase fluorescein angiogram reveals a progressive increase in the pigment epithelial leak with pooling of the subneurosensory retinal space. A small, irregular serous detachment of the retinal pigment epithelium in the nasal macula is also evident.

of the retinal pigment epithelial dysfunction remains unclear. Negi and Marmor ${ }^{12}$ believe a metabolic disturbance and failure of the retinal pigment epithelial cell ion pumping mechanism is responsible, whereas Spitznas ${ }^{13}$ argues that central serous chorioretinopathy occurs secondary to a reversal in the direction of ion pumping. If the composition of the subretinal exudative deposit is fibrin, a considerable alteration in the normal physiology of the choriocapillaris, Bruch's membrane, and the retinal pigment epithelium must occur. For fibrin to form on the undersurface of the retina, fibrinogen, the monomeric precursor of fibrin, must gain access to the subretinal space. Fibrinogen has a molecular weight of 343000 daltons, ${ }^{15}$ which is about five times greater than albumin, a protein shown to be restricted by the choriocapillaris. ${ }^{16}$

After resolution of the subretinal fluid and dissolution of the exudative deposit some patients had a permanent alteration in the appearance of the underlying retinal pigment epithelium. This altered appearance may be from residua of the subretinal exudative deposit, from healing of the underlying retinal pigment epithelial detachment, or from fibrous metaplasia of the retinal pigment epithelium. ${ }^{12}$

The 11 patients described in this report had central serous chorioretinopathy associated with

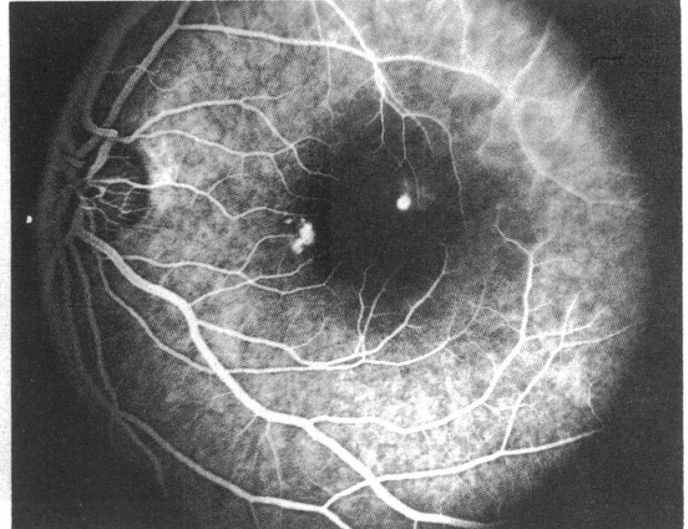

Figure $3(B)$ The early venous phase fluorescein angiogram reveals some window defect in the nasal macula and a focal leak at or near the edge of a serous detachment of the retinal pigment epithelium which is just superotemporal to the foveal region. This pigment epithelial detachment was clinically obscured by the exudate.

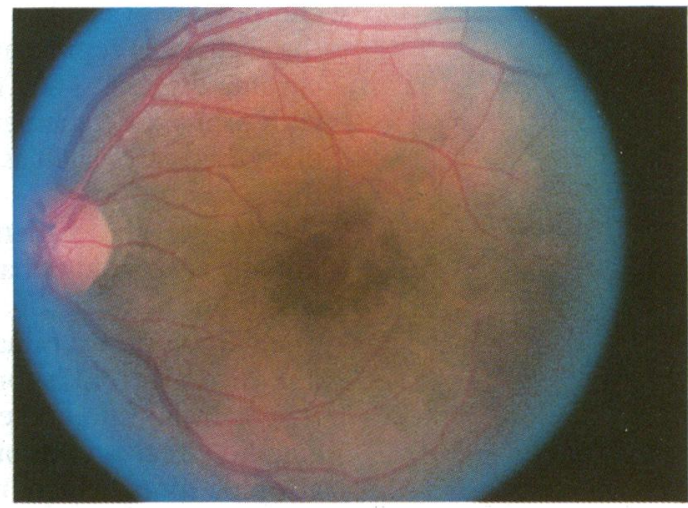

Figure 3(D) The same patient following laser photocoagulation treatment. Note the resolution of the neurosensory detachment and subretinal exudate.

subretinal deposition of material, which was believed to be proteinaceous and possibly fibrinous in nature. In contrast to previous reports, four non-pregnant women were noted in our series to have subretinal exudative deposits. The subretinal deposits were feather-edged, greyish white, translucent plaques overlying or closely associated with a leak at the level of the retinal pigment epithelium, which is not characteristic in appearance or location for a subretinal deposit associated with choroidal neovascularisation. The accompanying clinical and fluorescein angiographic findings, as well as the clinical course of these eyes, were consistent with the diagnosis of central serous chorioretinopathy. For protein, or more specifically fibrin, to be present in the subretinal space and on the outer surface of the retina, a breakdown in the normal physiology of the choriocapillaris, Bruch's membrane, and the retinal pigment epithelium must occur.

This work was supported in part by the LuEsther T Mertz Retinal Research Laboratory and the Macula Foundation, Manhattan Eye, Ear and Throat Hospital, 210 East 64th Street, New York, NY 10021, USA.

1 Gass JDM. Central serous chorioretinopathy and white subretinal exudation during pregnancy. Arch Ophthalmol 1991; 109: 677-81.

2 Gass JDM. Bullous retinal detachment: an unusual manifestation of idiopathic central serous choroidopathy. $\mathrm{Am} \mathcal{F}$ Ophthalmol 1973; 75: 810-21. 
3 Akiyama K, Kawamura M, Ogata T, Tanaka E. Retinal vascular loss in idiopathic central serous chorioretinopathy with bullous retinal detachment. Ophthalmology 1987; 94: 1605-9.

4 Yannuzzi LA, Shakin JL, Fisher YI, Altomonte MA. Peripheral retinal detachments and retinal pigment epithelial atrophic tracts secondary to central serous
pigment epitheliopathy. Ophthalmology 1984; 91: 1554pigment epitheliopathy. Ophthalmology 1984; 91: 1554

5 Urayama A, Hatakeyama T, Machida A, Abe N. Two cases of central chorioretinitis followed by retinal detachment. Fap $\mathcal{F}$ Clin Ophthalmol 1971; 25: 731-5.

6 Goldstein BG, Pavan PR. 'Blow outs' in the retinal pigment epithelium. Brf Ophthalmol 1987; 71: 676-81.

7 Krishan NR, Chandra SR, Stevens RS. Diagnosis and pathogenesis of retinal pigment epithelial tears. Am F Ophthalmo 1985; 100: 698-707

8 Ie D, Yannuzzi LA, Spaide RF, Woodward KP, Singerman LJ, Blumenkranz MS. Microrips of the retinal pigment epithelium. Arch Ophthalmol 1992; 110: 1443-9.

9 de Venecia G. Fluorescein angiographic smoke stack: case presentation at Verhoeff Society Meeting, Washington, DC 24-25 April, 1982.

10 Ikui $\mathrm{H}$. Histologic examination of central serous retinopathy. Nippon Ganka Kiyo 1969; 20: 1035-41.

11 Shimizu K, Tobari I. Central serous retinopathy dynamics of subretinal fluid. Mod Prob Ophthalmol 1971; 9: 152.

12 Negi A, Marmor M. Experimental serous retinal detachment and focal pigment epithelial damage. Arch Ophthalmol 1984; 102: 445-9.

13 Spitznas $M$. Pathogenesis of central serous retinopathy: a new working hypothesis. Graefes Arch Clin Exp Ophthalmol 1986; 224: 321-4.

14 Piccolino FC. Central serous chorioretinopathy: some considerations on the pathogenesis. Ophthalmologica 1981; 182 204-10.

15 Williams WJ, Beutler E, Ersleu A, Lichtman M. Hematology New York: McGraw-Hill, 1983: 1222-38.

16 Pino RM. Restriction to endogenous plasma proteins by fenestrated capillary endothelium: an ultrastructural immunocytochemical study of the choriocapillary endothelium. Am $\mathcal{F}$ Anatomy 1985; 172: 279-89. 